\title{
ESCARABAJOS (COLEOPTERA: BRUCHIDAE) ASOCIADOS A SEMILLAS DEL GÉNERO PISCIDIA L., 1759 (LEGUMINOSAE)
}

\section{SEED BEETLES (COLEOPTERA: BRUCHIDAE) ASSOCIATED TO THE GENUS PISCIDIA L., 1759 (LEGUMINOSAE)}

\author{
JESÚS ROMERO NÁPOLES ${ }^{1, *}$ \\ ${ }^{1}$ Posgrado en Fitosanidad-Entomología y Acarología, Colegio de Postgraduados, Montecillo, Estado de México, \\ km 36.5 carr. México-Texcoco, C.P. 56230. \\ * Autor de correspondencia: <jnapoles@colpos.mx> \\ Recibido: 08/03/2016; aceptado: 04/08/2016 \\ Editor responsable: Arturo Bonet Ceballos
}

Romero-Nápoles, J. (2016). Escarabajos (Coleoptera: Bruchidae) asociados a semillas del género Piscidia L., 1759 (Leguminosae). Acta Zoológica Mexicana (n.s.), 32(3), 286-295.

RESUMEN. Se presenta una clave para la identificación de cuatro especies de brúquidos que se alimentan de semillas de Piscidia spp.; Ctenocolum janzeni Kingsolver \& Whitehead, C. podagricus (Fabricius), C. pygospilotos Albuquerque \& Ribeiro-Costa y Stator monachus (Sharp); además se brinda información sobre el daño en semillas, plantas hospederas y su distribución, incluyendo nuevos registros.

Palabras clave: Escarabajos de las semillas, Bruchidae, Piscidia, plantas hospederas.

\section{INTRODUCCIÓN}

El género Piscidia (L.), 1759 incluye legumbres faboides que son usualmente arborescentes, se localizan principalmente en la parte norte de los neotrópicos; comúnmente conocidas como "envenenadoras de peces", fácilmente se les puede reconocer por sus frutos que presentan cuatro alas. El nombre común hace alusión a que nativos de Jamaica utilizaban la madera machacada de la planta y ésta era lanzada al río, donde se presumía la presencia de peces, al tiempo éstos eran intoxicados y sus cuerpos empezaban a flotar con sus abdómenes hacia arriba; si éstos no eran capturados, al poco tiempo se recuperaban. Peces capturados bajo este sistema se consideró un excelente alimento y a partir de este evento este grupo de plantas tomó interés, unido al hecho de que su madera se consideró una de las mejores en la isla (Rudd 1969). Los usos de estas plantas últimamente se han incrementado, por ejemplo Zamora et al. (2009) consideran que la especie Piscidia piscipula (L.) para el estado de Yucatán, México cuenta con los siguientes usos: medicinal, cons-
Romero-Nápoles, J. (2016). Seed beetles (Coleoptera: Bruchidae) associated to the Genus Piscidia L., 1759 (Leguminosae). Acta Zoológica Mexicana (n.s.), 32(3), 286-295.

ABSTRACT. A taxonomic key is presented in order to identify four bruchid species that feed on Piscidia spp. seeds: Ctenocolum janzeni Kingsolver \& Whitehead, C. podagricus (Fabricius), C. pygospilotos Albuquerque \& Ribeiro-Costa, and Stator monachus (Sharp); also information about the damage in seeds, host plants, and distribution, including new records are given.

Key words: Seed beetles, Bruchidae, Piscidia, host plants.

trucción, melífera, maderable, combustible, elaboración de cercos y forrajero. De acuerdo a la revisión de Rudd (1969), el género Piscidia está conformado por siete especies, de las cuales cuatro se encuentran en México; por esta razón se consideró como centro de dispersión al núcleo geológico que se localiza entre Guatemala y sur de México.

Las primeras noticias de la asociación entre brúquidos y plantas del género Piscidia fueron registradas por Zacher (1952), indicando a P p piscipula como hospedero de Ctenocolum podagricus. Posteriormente los siguientes autores hicieron valiosas aportaciones a la relación planta-insecto al indicar las especies de las semillas que son utilizadas como alimento por los brúquidos: Alburquerque et al. (2014), De la Cruz et al. (2013), Romero \& Johnson (2004), Turnbow et al. (2003), Johnson \& Siemens (1995), Johnson \& Nilsson (1990), Johnson et al. (1989), Hetz \& Johnson (1988), Johnson (1977, 1984), Janzen (1975, 1977, 1978, 1980), Terán \& Muruaga (1979), C.D. Johnson \& Kingsolver (1976), Kingsolver \& Whitehead (1974a), Kingsolver (1972) y Luca (1972). 
Dada la importancia de este grupo de plantas, los objetivos del presente estudio fueron elaborar una clave para la determinación de brúquidos asociados a plantas del género Piscidia, brindando una diagnosis de las especies, incluyendo información biológica, distribución y hospederos; finalmente se realiza un análisis del daño en las semillas de la especie $P$. piscipula ocasionado por los brúquidos.

\section{MATERIALES Y MÉTODOS}

Preparación de material entomológico. Para la preparación de las genitalias se utilizaron los métodos descritos por Kingsolver \& Whitehead (1974b) y Kingsolver (1970). Para la interpretación de las genitalias se siguió a Romero \& Johnson (1999). Se consultaron las siguientes colecciones para la revisión de especímenes: Colección Entomológica del Instituto de Fitosanidad, Colegio de Postgraduados, Montecillo, Estado de México (CEAM); Florida State Collection of Arthropods, Gainesville, FL, EE. UU. (FSCA); National Museum of Natural History, Smithsonian Institution, EE. UU. (USNM); Colección particular de Robert H. Turnbow (RHTC); Insect Collection Texas A\&M University, Department of Entomology, Texas, EE. UU. (TAMU); Hungarian Natural History Museum, Hungary (HNHM). Todas las plantas se citan de acuerdo con "The International Legume Database and Information Service ILDIS”. Para complementar la información sobre distribución y plantas hospederas de brúquidos se utilizó la base de datos BRUCOL (Romero \& Johnson, 2002).

Estimación de daño en semillas. Para la evaluación de daño se realizó la siguiente colecta de material: Km 57 autopista Veracruz- La Tinaja, Ver., 26/VI/2012, J. Romero N. col., criado de la semilla JRN \# 586/2012, Piscidia piscipula (L.) Sarg.

\section{RESULTADOS}

Clave para las cuatro especies de Bruchidae asociadas a semillas del género Piscidia.

1. Protórax no giboso, con carina lateral extendiéndose hasta la cavidad coxal; fémur posterior con sólo una espina; metatibia recta. . . . . . . . Stator monachus (Sharp) 1'. Protórax giboso, con carina lateral obsoleta, sin extenderse hasta la cavidad coxal; fémur posterior fuerte con 7-16 espinas formando un pecten y dos o más pequeños dientes antes del pecten; metatibia arqueada... Género Ctenocolum .......................... 2 2(1'). Fémur posterior con el segundo diente del pecten regular en tamaño hasta el ápice de la estructura en vista de perfil; tibia posterior con la superficie dorsal con dentículos; pigidio de la hembra cubierto de setas blancas, excepto por tres manchas obscuras laterales y una central..... . Ctenocolum janzeni Kingsolver \& Whitehead 2'. Fémur posterior con el segundo diente del pecten gradualmente incrementando de tamaño hasta la parte media de la estructura y decreciendo hacia el ápice; tibia posterior con la superficie dorsal sin dentículos; pigidio de la hembra con diferente patrón de manchas . ......... 3 3(2') Estrías elitrales con punturas impresas profundas; pigidio con la región media basal con punteaduras impresas profundas; élitros ligeramente variegados, con setas amarillo grisáceas y blancas; tercera interestría sin setas blancas en la base y en la región submediana con una banda pequeña de setas blancas ...Ctenocolum pygospilotos Albuquerque \& Ribeiro-Costa

3'. Estrías elitrales con punturas impresas poco profundas; pigidio con la región media basal con punteaduras impresas poco profundas; élitros fuertemente variegados, con setas cafés, negras y blancas; tercera interestría con una banda corta de setas blancas en la base y otra en la región submediana... Ctenocolum podagricus (Fabricius)

\section{Brúquidos asociados a semillas del género Piscidia}

Actualmente se encuentran registradas las siguientes cuatro especies que son capaces de alimentarse de semillas de especies de Piscidia: Ctenocolum janzeni, C. podagricus, C. pygospilotos y Stator monachus.

\section{Ctenocolum janzeni Kingsolver \& Whitehead, 1974}

Diagnosis. Longitud (pronoto-élitros) 3.1-4.1 mm; ancho 2.1-2.7 mm; máxima profundidad del tórax $2.4 \mathrm{~mm}$. Dorso con pubescencia variegada, con setas negras, rojizas y cafés. Pronoto con una giba media elevada, dividida por un surco longitudinal y transversal; gibas laterales fuertemente elevadas; lóbulo basal generalmente sin depresión y ligeramente o fuertemente emarginado. Estrías elitrales con punturas moderadamente impresas; base de estrías 3 y 4 con dientes poco conspicuos; diente de la estría 4 más cercano a la base del margen anterior del élitro que el diente de la estría 3; estría 6 conspicuamente impresa 


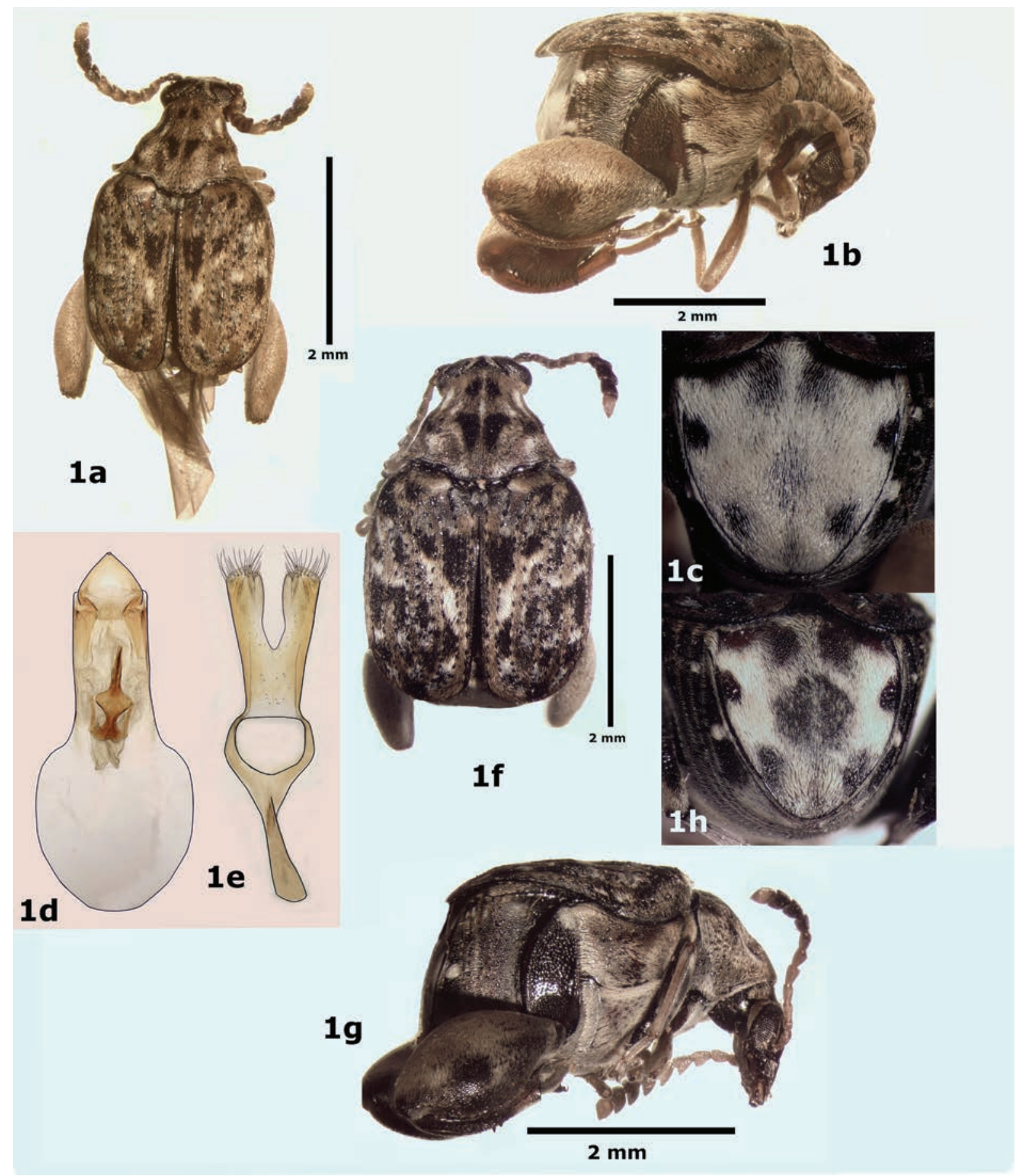

Figura 1. Ctenocolum janzeni, a) vista dorsal del macho, b) vista lateral del macho, c) pigidio del macho, d) lóbulo medio de la genitalia del macho, e) lóbulos laterales de la genitalia del macho, f) vista dorsal de la hembra, g) vista lateral de la hembra, h) pigidio de la hembra.

(Fig. 1a, b). Fémur posterior con el margen ventral externo con una carina dentada; pecten con 13-18 dientes. Pigidio más largo que ancho, oval, región media basal con punteaduras moderadamente impresas; cubierto con una pubescencia blanca excepto por cuatro pequeñas áreas glabras (Fig. 1c). Genitalia del macho como en la Figura 1d-e. Hembra similar al macho (Fig. 1f, g), excepto por el último esternito que no está emarginado y el 
pigidio que presenta un mayor número de áreas glabras (Fig. 1h).

Plantas hospederas. Lonchocarpus emarginatus Pittier, L. nitidus (Vogel) Benth., L. rugosus Benth., Piscidia carthagenensis Jacq., P. grandifolia (Donn. Sm.) I.M. Johnston, P. mollis Rose y P. piscipula (L.) Sarg. (Leguminosae).

Distribución. Costa Rica (Guanacaste, Puntarenas), Dominica (nuevo registro), Guadalupe, Guatemala (nuevo registro), Honduras (Olancho), México. (Jalisco, Morelos, Oaxaca, San Luis Potosí, Sinaloa, Sonora, Tamaulipas, Veracruz. Nuevos registros para los estados: Chiapas y Michoacán).

Material revisado. Dominica: St. John Par., Cabrits Nat. Park, 28/VI/2004, col. Turnbow R. (ex.1; CEAM). Guatemala: Suchitepéquez, Los Tarrales Reserve, 7/VI/2005, 1500 m, col. Turnbow R. (ex. 1; RHTC). México: Chiapas, El Sumidero, Mirador, La Coyota, 24/VI/1990, col. Turnbow R. (ex. 1; FSCA); Michoacán, km 167.5, Hwy.37, 32 km S Cuatro Caminos, 24/VII/2003, 230 m, col. Bellamy C.L. (ex. 1; CEAM).

\section{Ctenocolum podagricus (Fabricius, 1801)}

Diagnosis. Longitud (pronoto-élitros) 2.0-3.4 mm; ancho 1.4-2.1 mm; máxima profundidad del tórax 1.26-1.59 mm. Dorso variegado con pubescencia café obscura a negra y café rojiza o predominantemente negra. Pronoto con gibosidad media ligeramente elevada, dividida o no por un surco longitudinal y no dividida por un surco transversal; gibosidad lateral ligeramente a moderadamente elevada; lóbulo basal con o sin depresión y ligeramente emarginado. Estrías elitrales con punturas moderadamente impresas; estrías 3 y 4 con dientes menos conspicuos en la base; diente de la estría 4 más cercano a la base del diente de la estría 3 que al margen anterior del élitro; estría 6 conspicuamente impresa (Fig. 2a, b). Fémur posterior con el margen ventral externo con carina dentada; sin dentículos arriba del margen ventral externo; pecten con 7-9 dientes. Pigidio más largo que ancho, región basal media con punturas moderadamente impresas (Fig. 2c). Genitalia del macho como en la Figura 2 d-e. Hembra similar al macho (Fig. 2f, g), excepto por el último esternito que no está emarginado y el pigidio de forma subtriangular y la pubescencia ligeramente diferente (Fig. 2h).

Plantas hospederas. Lonchocarpus costaricensis (Donn. Sm.) Pittier, L. eriocarinalis Micheli, L. heptaphyllus (Poir.) Dc., L. hondurensis Benth., L. margaritensis Pit- tier, L. minimiflorus Donn. Sm., L. muehlbergianus Hassl., L. nitidus (Vogel) Benth., L. parviflorus Benth., L. rugosus Benth., Piscidia carthagenensis Jacq., y P. piscipula (L.) Sarg. (Leguminosae).

Distribución. Argentina (nuevo registro), Bolivia (nuevo registro), Brasil (Mato Grosso Paraná, Sao Paulo. Nuevo registro para el país: Rondonia), Costa Rica (Guanacaste, Puntarenas), Cuba, Ecuador (Napo), El Salvador (La Unión, San Salvador), Guatemala (nuevo registro), Guyana, Jamaica (nuevo registro), México (Campeche, Chiapas, Jalisco, Querétaro, Quintana Roo, Sinaloa, Tabasco, Veracruz), Nicaragua, Paraguay (nuevo registro), Perú (nuevo registro), Puerto Rico, St. Barthelemy, Trinidad \& Tobago, Venezuela (Distrito Federal).

Material revisado. Argentina: Iguazú, Misiones, XI/1993, Peña L. (ex 1FSCA). Bolivia: Santa Cruz, 3.7 km SSE Buena Vista, Hotel Flora \& Fauna, 15/XI/2001, 430 m, Thomas M.C., 6333.152’ O, 17²9.949’ S (ex 1 FCSA); Santa Cruz, 3.7 km SSE Buena Vista, Hotel Flora \& Fauna, 19/X/2000, 430 m, Thomas M.C., (ex 1 FCSA); Santa Cruz, 3.7 km SSE Buena Vista, Hotel Flora \& Fauna, 28/X/2000, Dozier B.K. (ex 1 FCSA); Santa Cruz, 4-6 km SSE Buena Vista, F\&F Hotel, 31/X/2002, Wappes \& Morris (ex 1 CEAM, ex 1 FCSA); Santa Cruz, 5 km SSE Buena Vista, Hotel Flora \& Fauna, 22/X/2004, Eger J.E., 63³9.128' O, 17²9.925’ S, (ex 2 FCSA); Santa Cruz, Buena Vista vic, Hotel Flora \& Fauna, 20/X/2000, Morris R.,(ex 1 FCSA); Santa Cruz, Potrerillos del Guendá, 40 km NW Santa Cruz de la Sierra, 20/XI/2004, Dozier B.K., 6327.44' O, 17040.265' S (ex 5 FCSA). Brasil: Rondonia, 62 km SW Ariquemes, nr. Fzda. Rancho Grande, 16/XI/1997, Eger J.E., en blacklight trap, (ex 2 FSCA); 62 km SW Ariquemes, nr. Fzda. Rancho Grande, 20/XI/1994, Eger J.E., en blacklight trap (ex 4 FSCA). Guatemala: Baja Verapaz, 15 km N Salama, 5/VI/1991, D Wappes J. E. (1 ex FCSA). Jamaica: Saint James, Montego Bay, 3/VIII/1967, O’Brien C.W. (ex 1 TAMU). Paraguay: Puerto P. Stroessner, 29/XII/1966, col. Mahunka (ex 1 HNHM).

\section{Ctenocolum pygospilotos Albuquerque \& Ribeiro- Costa, 2014}

Diagnosis. Longitud (pronoto-élitros) $2.8 \mathrm{~mm}$; ancho 2.0 mm; máxima profundidad del tórax $1.73 \mathrm{~mm}$. Dorso con pubescencia ligeramente variegada de color café rojizo y negro. Pronoto con gibosidad media ligeramente elevada, no dividida por un surco longitudinal y transverso; gibo- 


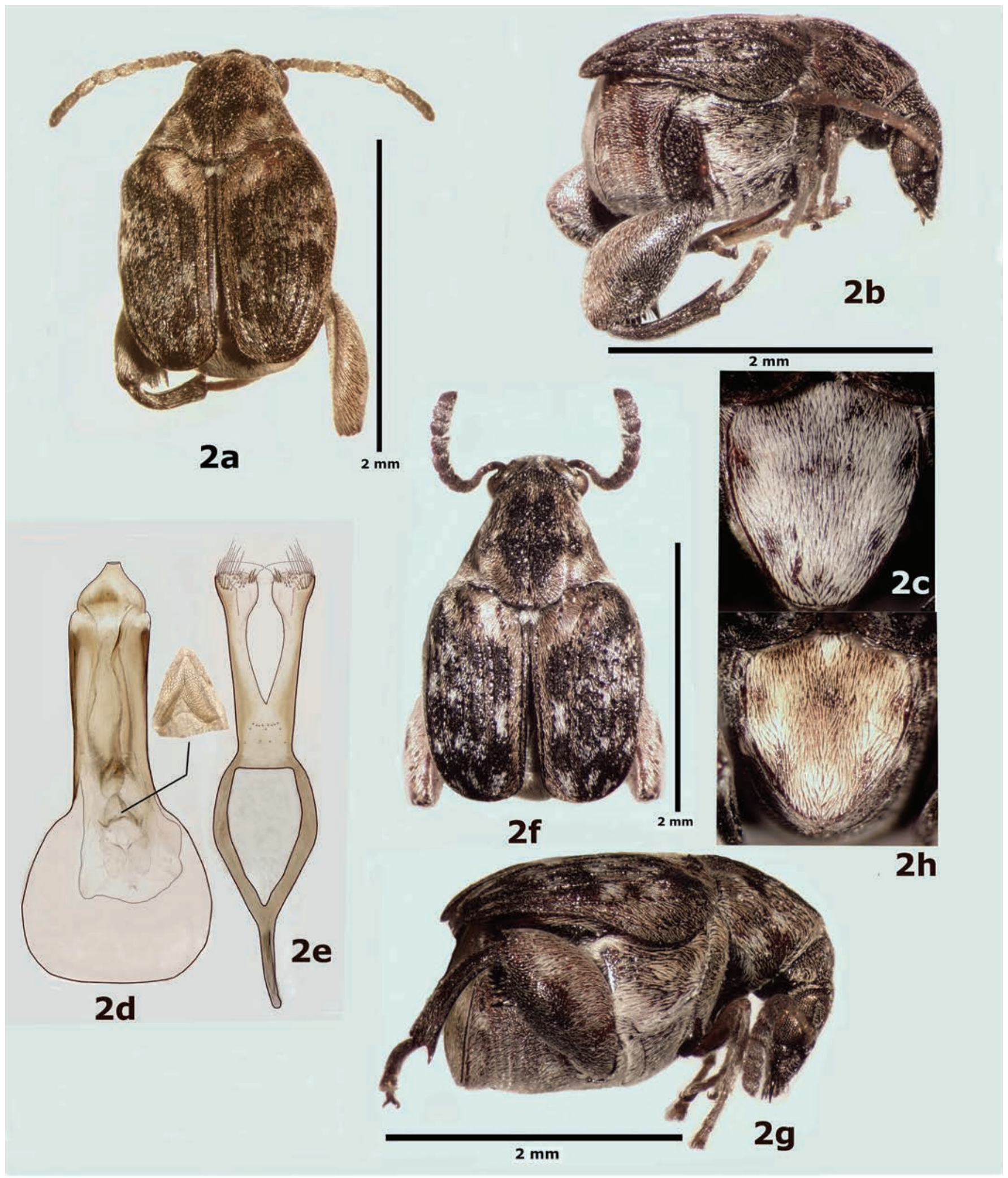

Figura 2. Ctenocolum podagricus, a) vista dorsal del macho, b) vista lateral del macho, c) pigidio del macho, d) lóbulo medio de la genitalia del macho, e) lóbulos laterales de la genitalia del macho, f) vista dorsal de la hembra, g) vista lateral de la hembra, h) pigidio de la hembra. 
sidad lateral ligeramente elevada; lóbulo basal con una depresión y ligeramente emarginado. Estrías elitrales con punturas impresas profundas; dientes en la base de la estría 3 y 4 menos conspicuos; diente de la estría 4 más cercano a la base del diente de la estría 3 que al margen anterior del élitro; estría 6 impresa conspicuamente (Fig. 3a, b). Fémur posterior con el margen externo ventral con carina dentada; sin dentículos arriba del margen externo ventral; pecten con 8 dientes. Tibia posterior fuertemente emarginada en la base adyacente al mucro; dentículos coronales laterales presentes. Pigidio más largo que ancho, oval, región basal media con punturas impresas profundas (Fig. 3c). Genitalia del macho como en la Figura 3d-e. Hembra desconocida.

Plantas hospederas. Piscidia piscipula (L.) Sarg. (Leguminosae).

Distribución. Estados Unidos (Islas Vírgenes).

\section{Stator monachus (Sharp, 1885)}

Diagnosis. Longitud (pronoto-élitros) 1.7-2.5 mm; ancho 1.2-1.8 mm; máxima profundidad del tórax 1.0-1.3 mm. Cabeza con el integumento negro, mandíbulas y palpos castaños, antenas con los segmentos basales amarillentos y el resto castaño, tórax negro, élitros negros cada uno con dos manchas castaño rojizas que no tocan los bordes; pata protorácica con coxa y trocánter castaños y el resto amarillento castaño; pata mesotorácica con coxa, trocánter, fémur y $1 / 3$ basal de la tibia castaño obscuro y el resto castaño amarillento; pata metatorácica negra; abdomen negro. Existen formas que son completamente melánicas y al parecer las manchas rojas de los élitros no están asociadas al sexo. Pubescencia blanquecina, distribuida más o menos uniformemente. Cabeza con carina frontal apenas marcada, élitro con estrías finas, poco profundas, con algunas punturas circulares más anchas que ellas; estría 1 y 2 llegan a la base, 3-6 se originan un poco antes del borde basal y la 7 detrás del húmero, 8 y 9 al costado de éste, la 10 se curvea hacia el ángulo latero-posterior del pronoto (Fig. 4a, b). Fémur posterior constreñido basal y apicalmente, expandido medialmente y ligeramente más ancho que la metacoxa, armado con una espina subapical tan larga como la anchura de la base de la metatibia. Pigidio subtriangular (Fig. 4c). Genitalia del macho como en la Figura 4d-e. Hembra similar al macho (Fig. 4f, g, h), excepto por el último esternito que no está emarginado distalmente.

Plantas hospederas. Acacia aff., glomerosa, A. bonariensis Hook. \& Arn., A. diversifolia (Schldl.) Benth.,
A. furcatispina Burkart, A. hayesii Benth., A. mearnsii De Wild., A. melanoxylon R. Br., A. polyphylla DC., A. praecox Griseb., A. riparia Kunth, A. tamarindifolia (L.) Willd., Cercidium praecox subsp. glaucum (Cav.) Burkart \& Carter, Parkinsonia praecox (Ruiz \& Pav. Ex Hook.) J. Hawkins, Piscidia piscipula (L.) Sarg., y Acacia podalyriifolia G. Don como un nuevo registro de hospedero (Leguminosae).

Distribución. Antigua \& Barbuda, Argentina (Buenos Aires, Catamarca, Corrientes, Córdoba, Entre Ríos, Formosa, La Rioja, Santa Fé, Tucumán), Bolivia (Santa Cruz, Tarija), Brasil (Bahía, Mato Grosso, Rio Grande do Sul, Rondonia), Colombia (Cundinamarca), Estados Unidos (Islas Vírgenes), Guadalupe, Guatemala (Jutiapa, Suchitepéquez), Honduras (Olancho), Islas Vírgenes Británicas, México (Nayarit, Veracruz), Panamá (Chiriquí, Panamá), Paraguay (Guariá), Puerto Rico, Región Reunión, San Cristóbal y Nieves, San Vicente y Las Granadinas, Uruguay (Canelones, Matarrita, Montevideo) y Venezuela (Anzoategui, Bolívar, Falcón, Lara, Zulia).

Material revisado. Uruguay: Matarrita (Intercepted at quarantine station, EE. UU.), 11/X/1958, reared seed Acacia podalyriifolia G. Don (ex 6 TAMU, ex 7 CEAM).

Estimación de daño en semillas. De un total de 506 semillas, 425 de ellas estuvieron sanas y 81 semillas presentaron un orificio de salida del brúquido o bien un orificio irregular, lo que denotó la emergencia de parasitoides; para este caso de evaluación de daño se consideró como semilla no viable. Los resultados del análisis indicaron que se presentó un porcentaje de daño de $19.05 \%$ causado por C. janzeni y C. podagricus en las semillas de $P$. piscipula, con una proporción de $64.8 \%$ y $35.2 \%$, respectivamente. Vale la pena comentar que las semillas de esta planta sólo pudieron alimentar a un insecto por semilla, sea cualquiera de las dos especies que haya tenido primeramente acceso al recurso.

Es interesante indicar que el hospedero primario de $C$. janzeni es Lonchocarpus rugosus Benth. (Leguminosae: Papilionoideae), según Romero (2002) los especímenes que emergen de las semillas de esta planta en promedio pueden medir en longitud $4.1 \mathrm{~mm}$ (pronoto-élitros), 2.7 mm de ancho y profundidad torácica $2.4 \mathrm{~mm}$; sin embargo, en condiciones de confinamiento y altas densidades la talla de los insectos disminuye casi en un 50\%, llegando a medir $2.2 \mathrm{~mm}$ de longitud, $1.5 \mathrm{~mm}$ de ancho y 1.4 de profundidad torácica. Este fenómeno de plasticidad genética permite que al reducir la especie su tamaño corporal está capacitada para invadir nuevos hospederos, por ejemplo 


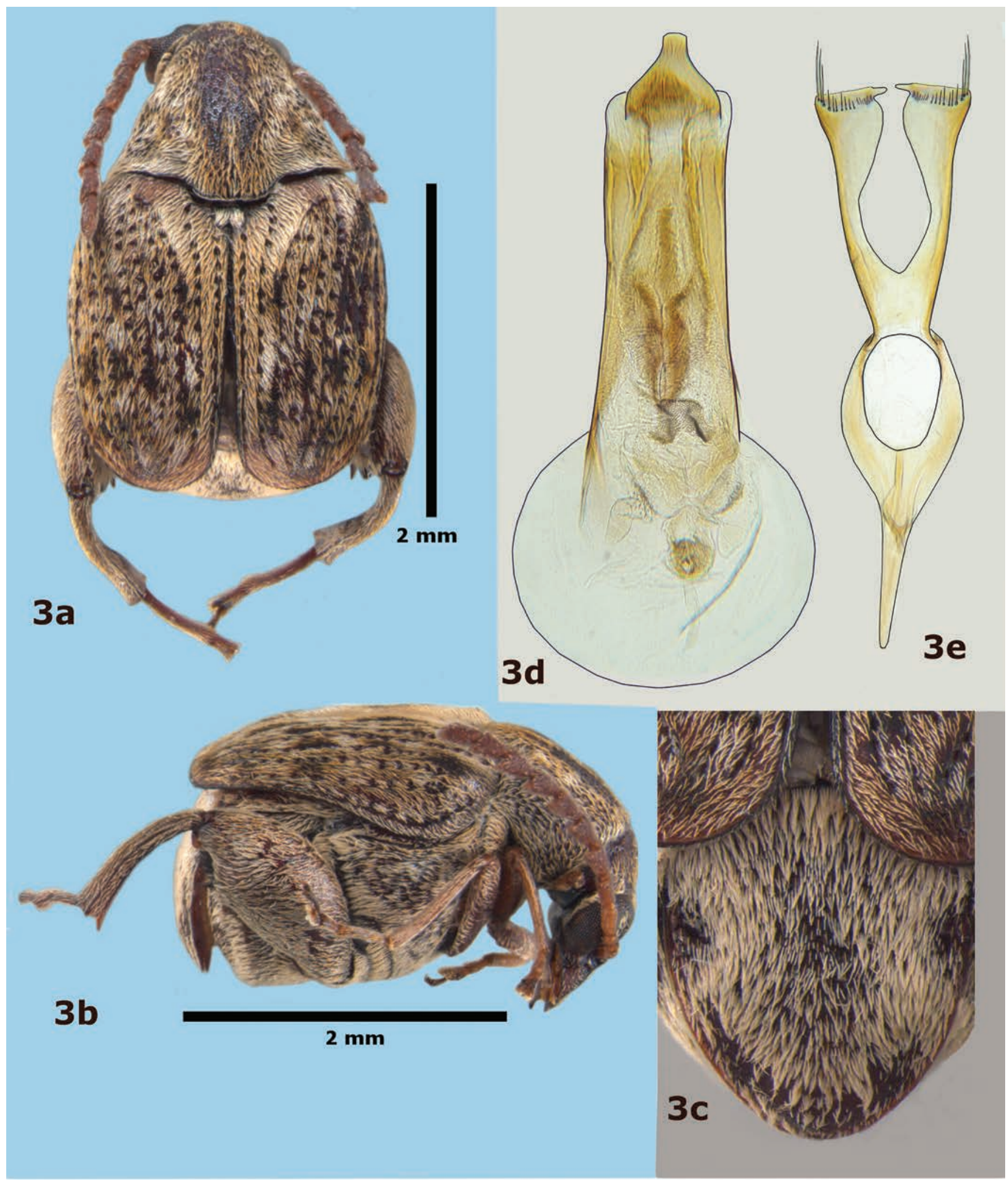

Figura 3. Ctenocolum pygospilotos, a) vista dorsal del macho, b) vista lateral del macho, c) pigidio del macho, d) lóbulo medio de la genitalia del macho, e) lóbulos laterales de la genitalia del macho. 

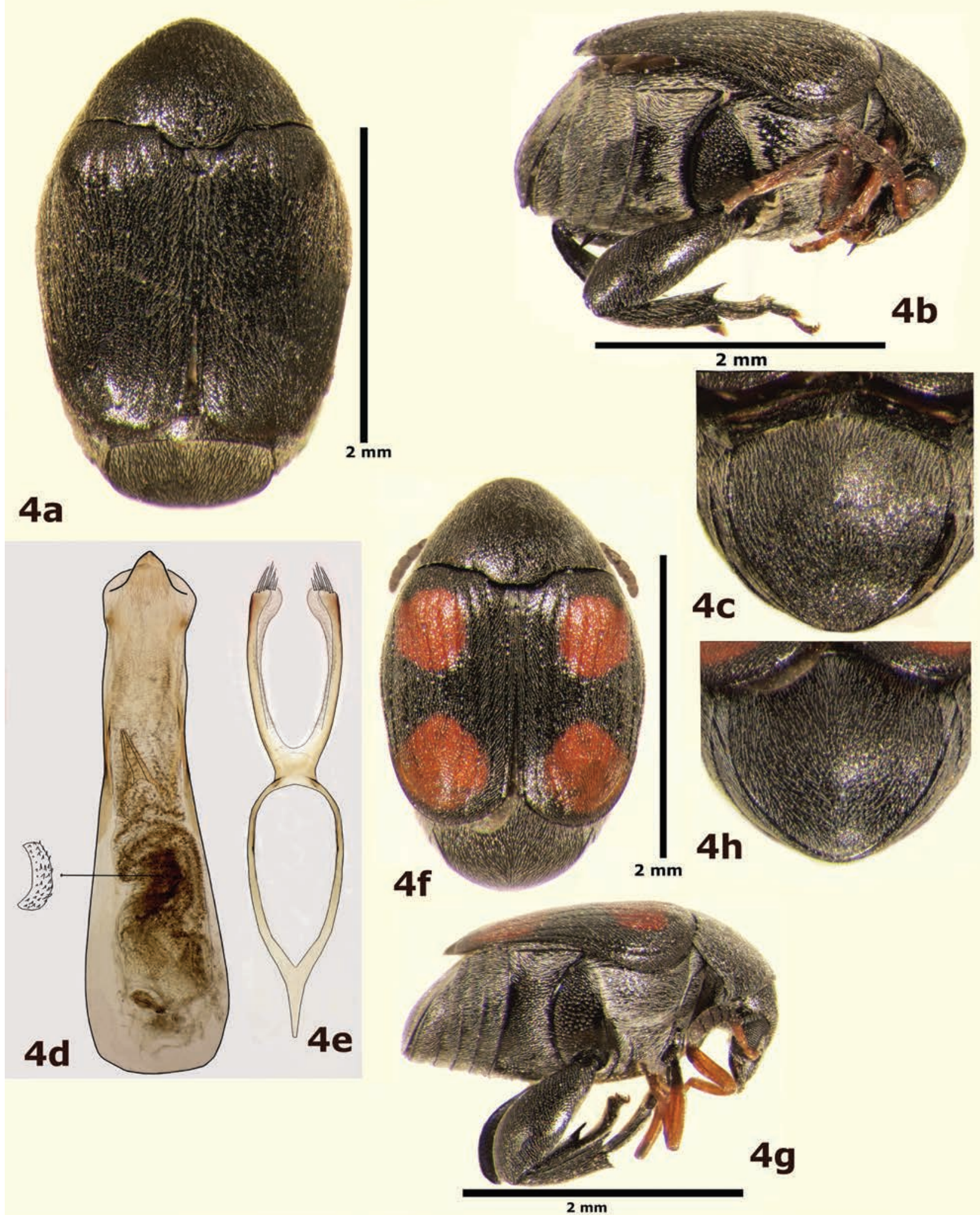

Figura 4. Stator monachus, a) vista dorsal del macho, b) vista lateral del macho, c) pigidio del macho, d) lóbulo medio de la genitalia del macho, e) lóbulos laterales de la genitalia del macho, f) vista dorsal de la hembra, g) vista lateral de la hembra, h) pigidio de la hembra. 
plantas con semillas más pequeñas, como es el caso de $P$. piscipula. Esta adaptación ecológica no es nueva en este grupo de insectos; al respecto Center \& Johnson (1974) y Johnson (1970) indican que Acanthoscelides aureolus (Horn, 1973) puede variar de tamaño, las formas pequeñas (1.1 mm de longitud) se pueden alimentar de semillas pequeñas del hospedero Syrmatium glabrum Vogel (=Lotus scoparius), en tanto que las grandes ( $2.7 \mathrm{~mm}$ de longitud) pueden utilizar semillas de mayor tamaño como las de Astragalus sp., o Glycyrrhiza lepidota. Kingsolver \& Whitehead (1974) ya habían indicado la presencia de Ctenocolum janzeni y C. podagricus compartiendo el mismo recurso (Piscidia carthagenensis Jacq.) pero en Costa Rica.

AGRADECIMIENTOS. A Felícia Pereira De Albuquerque del Laboratorio de Sistemática y Bioecología de Coleoptera, Departamento de Zoología, Universidad Federal de Paraná, Brasil por permitirme utilizar las imágenes de Ctenocolum pygospilotos.

\section{LITERATURA CITADA}

Alburquerque, F. P., Manfio, D., \& Ribeiro, C. C. S. (2014). A contribution to the knowledge of New World Bruchinae (Coleoptera, Chrysomelidae): taxonomic revision of Ctenocolum Kingsolver $\&$ Whitehead, with description of five new species. Zootaxa, 3838(1), 1-45.

Center, T. D., \& Johnson, C. D. (1974). Coevolution of some seed beetles (Coleoptera: Bruchidae) and their hosts. Ecology, 55, 1096-1103.

De la Cruz, P. A., Romero, N. J., Carrillo, S. J. L., García E. L., Grether, G. R., Sánchez, S. S., \& Pérez, de la C. M. (2013). Brúquidos (Coleoptera: Bruchidae) del estado de Tabasco, México. Acta Zoológica Mexicana (n.s.), 29(1), 1-95.

Hetz, M., \& Johnson, C. D. (1988). Hymenopterous parasites of some bruchid beetles of North and Central America. Journal of Stored Products Research, 24(3), 131-143.

Janzen, D. H. (1975). Interactions of seeds and their insects predators/ parasitoids in a tropical deciduous forest, pp.154-186. In: P.W. Price (Ed.). Evolutionary Strategies of Parasitic Insects and Mites. Plenum Press, New York.

Janzen, D. H. (1977). The Interaction of Seed Predators \& Seed Chemistry, pp. 415-428. In: V. Labeyrie (Ed.). Comportement des insectes et milieu trophique. Colloque Internationaux du C.N.R.S., Paris. 493 p.

Janzen, D. H. (1978). The Ecology and Evolutionary Biology of seed Chemistry as Relates to Seed Predation, pp. 163-206. In: J. B. Harborne, (Ed.). Biochemical Aspects of Plant and Animal Coevolution. Academic Press, London.

Janzen, D. H. (1980). Specificity of seed-attacking beetles in a Costa Rican deciduous forest. Journal of Ecology, 68, 929-952.

Johnson, C. D. (1970). Biosystematics of the Arizona, California and Oregon Species of the Seed Beetle Genus Acanthoscelides Schils- ky (Coleoptera: Bruchidae). University of California Publications in Entomology, 59, 1-116.

Johnson, C. D. (1977). Life history of Ctenocolum janzeni (Coleoptera: Bruchidae) in seeds of Piscidia mollis (Leguminosae). The Coleopterists Bulletin, 31(4), 313-318.

Johnson, C. D. (1984). New host records and notes on the biology of Stator (Coleoptera: Bruchidae). The Coleopterists Bulletin, 38(1), 85-90.

Johnson, C. D., \& Kingsolver, J. M. (1976). Systematics of Stator of North and Central America (Coleoptera: Bruchidae). USDA Technical Bulletin, 1537. $101 \mathrm{p}$.

Johnson, C. D., Kingsolver, J. M., \& Terán, A. L. (1989). Sistemática del género Stator en Sudamérica (Insecta: Coleoptera: Bruchidae). Opera Lilloana, 37, 1-105.

Johnson, C. D., \& Nilsson, J. A. (1990). New synonymy of Bruchus podagricus Fabricius and Bruchus crotonae Fahraeus, with a lectotype designation and a new combination for B. podagricus (Coleoptera: Bruchidae: Ctenocolum). Pan-Pacific Entomologist, 66(2), 171-172.

Johnson, C. D., \& Siemens, D. H. (1995). Bruchid guilds, host preferences, and new host records from Latin America and Texas for the genus Stator Bridwell (Coleoptera: Bruchidae). The Coleopterists Bulletin, 49(2), 133-142.

Kingsolver, J. M. (1970). A study of male genitalia in Bruchidae (Coleoptera). Proceedings of the Entomological Society of Washington, 72, 370-386.

Kingsolver, J. M. (1972). Synopsis of the genus Stator Bridwell in the West Indies, with descriptions of new species. Proceedings of the Entomological Society of Washington, 74(2), 219-229.

Kingsolver, J. M., \& Whitehead, D R. (1974a). Biosystematics of Central American species of Ctenocolum, a new genus of seed beetles (Coleoptera: Bruchidae). Proceedings of the Entomological Society of Washington, 87, 283-312.

Kingsolver, J. M., \& Whitehead, D. R. (1974b). Classification and comparative biology of the seed beetle genus Caryedes Hummel (Coleoptera: Bruchidae). Transactions of the American Entomological Society (Philadelphia), 100, 341-436.

Luca, Y. de. (1972). Catalogue raisonné des insectes Antilles Francaises. Annales de Zoologie-Écologie animale, 4(2), 103-107.

Romero, N. J. (2002). Bruchidae, pp. 513-534. In: Bousquets, J. L. \& Morrone, J. J. (Eds.) Biodiversidad, Taxonomía y Biogeografía de Artrópodos de México: Hacia una síntesis de su conocimiento. Vol. III. D. R. Universidad Nacional Autónoma de México.

Romero, J., \& Johnson, C. D. (1999). Zabrotes sylvestris, a new species from the United States and Mexico related to Z. subfasciatus (Boheman) (Coleoptera: Bruchidae: Amblycerinae). The Coleopterists Bulletin, 53, 87-98.

Romero N., J., \& Johnson, C. D. (2002). Date Base BRUCOL. Programa de Entomología, Instituto de Fitosanidad, Colegio de Postgraduados, México.

Romero, N. J., \& Johnson, C. D. (2004). Checklist of the Bruchidae (Coleoptera) of Mexico. The Coleopterists Bulletin, 58(4), 613635.

Rudd, V. E. (1969). A synopsis of the genus Piscidia (Leguminosae). Phytologia, 18(8), 473-499.

Terán, A. L., \& Muruaga, de L. A. S. (1979). Observaciones sobre Bruchidae (Coleoptera) del noroeste Argentino II. Estudios 
morfológicos y biológicos de algunas especies de Amblycerinae y Bruchinae. Acta Zoologica Lilloana, 35, 435-474.

Turnbow, R. H., Ronald, D. C., \& Kingsolver, J. M. (2003). An annotated checklist of the Bruchidae of Honduras. Ceiba, 44(2), 269-278.
Zacher, F. (1952). Die Nahrpflanzen der Samenkafer. Zeitschrift fuer Angewandte Entomologie, 33, 460-480.

Zamora, C. P., Flores G. J. S., \& Ruenes, R. M. (2009). Flora útil y su manejo en el cono sur del estado de Yucatán, México. Polibotánica, 28, 227-250. 